\title{
Dynamic trial fitting of the cup in press-fit total hip arthroplasty, a feasibility study
}

\author{
Daniël Hoornenborg, Justin Van Loon, Sheryl De WaArd, Inger N. Sierevelt, Kim T.M. Opdam, \\ Gino M.M.J. Kerkhoffs, Daniël HaVerkamp
}

From the Xpert Clinics Orthopedie, Amsterdam, The Netherlands

Trial fitting of the cup during total hip arthroplasty (THA) is done by trial cups, which do not resemble the real press-fit obtained by the definitive implant. Our goal is to judge feasibility of the $\mathrm{X}$-pander ${ }^{\circledR}$ in clinical practice; a device developed to mimic the real press-fit obtained by the definitive cup, to ensure satisfactory press-fit.

In this feasibility study 45 experienced orthopaedic surgeons from 7 European countries filled in a structured survey after 78 primary THA and 31 revision surgeries, using the $X$-pander instead of traditional trial cups. Primary outcomes were decision change concerning cup size or further reaming and increased confidence regarding cup insertion and size. Additionally, potential association between the primary outcomes and procedure (primary or revision), bone quality and experience of the surgeon were evaluated.

In $33.3 \%$ of the primary and $32.2 \%$ of the revision cases the $\mathrm{X}$-pander measurement changed the decision and further reaming or change of cup size was decided. In $61.5 \%$ and $58.1 \%$ of respectively the primary and revision THAs the $\mathrm{X}$-pander was judged to give fairly to much more reliable information than traditional trial cups. The $\mathrm{X}$-pander could lead to less additional screw fixation, as stated in $37.2 \%$ of the primary and $25.8 \%$ of the revision cases and to better cup insertion in respectively $50.0 \%$ and $51.6 \%$.

This study validates that the $\mathrm{X}$-pander may be a suitable option for accurate sizing and assessment of the reamed acetabulum and could replace traditional trial cups in THA.

No benefits or funds were received in support of this study. None of the authors have a conflict of interest.
Keywords : press-fit ; trial fitting ; acetabular component ; total hip arthroplasty.

\section{INTRODUCTION}

When placing a press-fit acetabular component during total hip arthroplasty (THA) it is important to ensure primary stability in order to secure transition to secondary stability by bone-ingrowth (1). For most of the designs of acetabular components this principle is well proven $(2,3)$. However, every implant has a specific learning curve, since the

Daniël Hoornenborg ${ }^{1}$, MD

Justin Van Loon ${ }^{1,2,3}$, MD

Sheryl De Waard ${ }^{1}, \mathrm{MD}$

Inger N. Sierevelt ${ }^{1}$, MSc

- Kim T.M. Opdam², MD

- Gino M.M.J. Kerkhoffs ${ }^{2}$, Prof. MD, PhD

- Daniël Haverkamp ${ }^{1}, \mathrm{MD}, \mathrm{PhD}$

${ }^{\prime}$ Xpert Clinics Orthopedie, Amsterdam, The Netherlands.

${ }^{2}$ Amsterdam University Medical Centres, location Academic Medical Center, department of Orthopaedic Surgery, Amsterdam, The Netherlands.

${ }^{3}$ Tergooi, department of Orthopaedic Surgery, Hilversum, The Netherlands.

Correspondence : Daniël Haverkamp, Laarderhoogtweg 12, 1101 EA Amsterdam, The Netherlands.

Email : d.haverkamp@xpertclinics.nl

- 2021, Acta Orthopædica Belgica.

Acta Orthopædica Belgica, Vol. 87 - 2 - 2021 
achieved press-fit is estimated on trial cups which do not have the same size as the implant and differ between brands of implants (4). Therefore, experience of the surgeon and familiarity with the implant is an important factor. The role of primary stability on outcome of THA is well documented and not an issue of debate $(1,5)$. However, the exact amount of sufficient press-fit is not clear and is currently based on the experience of the surgeon (3). Achieving the ideal primary stability remains a well-educated guess, especially since the actual press-fit is not exactly mimicked by the current design of trial cups.

In case of revision surgery of the acetabular component primary press-fit is equally relevant (67). However, assessment of initial press-fit by trial cups is more difficult and requires more surgical experience due to variations in bone geometry and mechanical properties. Especially in case of large defects or poor bone quality. The decision whether press-fit can be obtained with an uncemented acetabular component can be difficult to make and often results in the choice to use a cemented cup. Despite the discussion which revision implant is superior, it would be helpful for the orthopaedic surgeon to have a more tactile feedback of the achieved press-fit with the uncemented implant, especially if this was his first choice for the selected case.

A recently developed tool to provide tactile feedback of initial press-fit is the X-pander ${ }^{\circledR}$. This tool is designed as a form of dynamic press-fit in which a trial fit cup can be expanded to mimic the stability and real size of the definitive cup (8). Moreover, the surface texture and shape are more comparable to the definitive cup than normal trial cups. After reaming during total hip replacement, a X-pander measuring head is fitted in to the acetabulum and can be expanded up to $3 \mathrm{~mm}$ until satisfactory fixation is achieved. This allows the surgeon to experience the real amount of pressfit to be obtained with the final implant. Hereby the surgeon can overcome both over- and underreaming of the acetabulum and can make a more objective decision for the size of the definitive cup based on the feeling of primary stability when using the X-pander.
The goal of this study is to investigate feasibility of the X-pander in clinical practice in terms of decision-making during surgery, confidence, usefulness and safety, in the hands of experienced hip surgeons, acquainted with THA.

\section{MATERIAL AND METHODS}

Forty-five surgeons in 7 European countries (Denmark, The Netherlands, France, Germany, Norway, Switzerland and the UK) and 20 Hospitals were asked to participate in this initial feasibility study. The participating orthopaedic surgeons were asked to use the X-pander in a series of primary and or revision cases and to fill in a carefully developed questionnaire after each procedure. The design and reporting were performed in accordance to the Strengthening the Reporting of OBservational studies in Epidemiology (STROBE) statement.

Since the X-pander has been introduced on the Market and has a CE marking, no IRB approval was required for this surgeon's survey.

The surgical approach was according to the preference of the orthopaedic surgeon and preparation of the acetabulum was according to the standard principles of acetabular cup placement. Trial fitting was done with the X-pander, a device developed by Medicahnical Engineering ApS. (Fig. 1) The measuring head is a single use device that is inserted in the acetabulum and can by expanded up to three $\mathrm{mm}$ in diameter by turning the knob of the handle. This device is available in 10 individual sizes with two mm increments, covering a span from

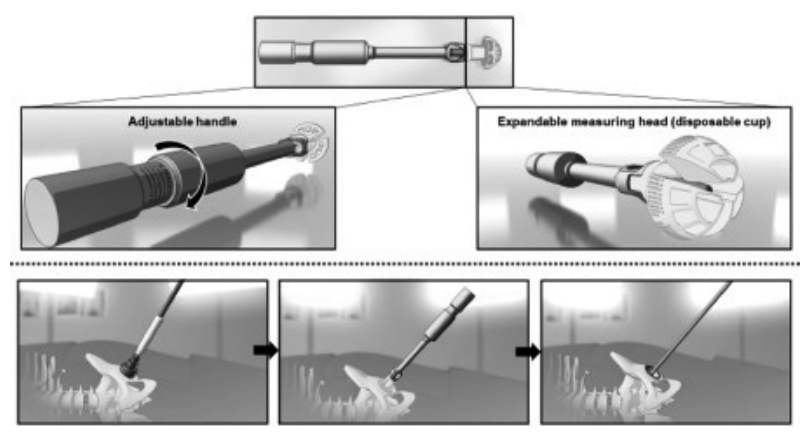

Fig. 1. - X-pander, by turning the handle the diameter of the $\mathrm{X}$-pander changes. The correct diameter can be read from the handle. (small pictures: reaming, X-pander testing and cup insertion). 
$48 \mathrm{~mm}$ to $69 \mathrm{~mm}$. For instance, if a $54 \mathrm{~mm}$ reamer is used and the true size of the implant is $55.4 \mathrm{~mm}$, then a $54 \mathrm{~mm}$ X-pander can be inserted and expanded for $1.4 \mathrm{~mm}$ to mimic the true press-fit of the definitive implant. If necessary, additional reaming can be done afterwards and the acetabular component matching the measured size that provides sufficient primary stability can be implanted.

Main questions of the survey concerned change of decision of the cup size, decision to ream further, and confidence regarding cup insertion and size. Supplementary questions regarding the use of the $\mathrm{X}$-pander were included : did a fracture occur, was the cup fully seated, and did you have any drawbacks while using the X-pander? Patient specific data (age, indication, gender, BMI), experience of the surgeon and operative details (bone quality: soft, medium or hard ; implant type ; size of the implant ; surgical approach; fixation methods of the cup) were also registered. The final questions consist of impression and opinions regarding usefulness of the $\mathrm{X}$-pander. The survey for revision procedures was identical to the primary procedure with additional specific questions regarding bone stock/defects and bone grafting. Missing data were obtained by contacting the orthopaedic surgeon that filled in the questionnaire.

The primary outcomes were 1) decision change concerning cup size, 2) decision for further reaming and 3) increased confidence regarding cup insertion and size.

As secondary outcome potential association between the primary outcomes and procedure (primary or revision), bone quality (soft, medium, hard) and experience of the surgeon were evaluated.

Statistical analyses were performed with Statistical Package for Social Sciences (SPSS) version 26.0 (SPSS Inc. Chicago, IL). Distribution of continuous variables was assessed using the Shapiro-Wilk tests. Normally distributed variables are stated as means with standard deviations (SD). Categorical data are described as numbers with accompanying proportions. To assess the association between the primary outcomes and procedure (primary or revision), bone quality (soft, medium, hard) and experience of the surgeon, mixed logistic regression analyses were performed to account for the correlation between multiple surveys within observers. A p-value of $<0.05$ was considered statistically significant.

This study was financially supported by Medichanical Engineering ApS. They were not involved in the determination of the study design; in the collection, analysis and interpretation of the data; in writing of the report or in the decision to submit the article for publication.

\section{RESULTS}

In this study 45 orthopaedic surgeons (109 cases) filled out the survey. The surgeons performed 78 primary procedures and 31 revision surgeries. Patients undergoing primary THA were mainly female (62.8.\%), had a mean age of 66.3 (SD 12.8), mean BMI of 28.1 (SD 6.0). The 31 revision cases were mainly female $(58.1 \%)$, with a mean age of 72.1 (SD 11.8) and mean BMI of 27.3 (SD 5.1).

In the primary cases ( 78 cases and 39 surgeons) the surgeon judged that the X-pander yields fairly to much more reliable information than traditional trial cups in $89.7 \%$. In $26(33.3 \%)$ of the procedures further reaming or change of cup size was done because of the X-pander. In $13(16.6 \%)$ procedures this was a combination of further reaming and change of cup-size, in five $(6.4 \%)$ procedures only additional reaming was done without change of cup size and in eight $(10.3 \%)$ cases only the size of the definitive implant was changed based on the $\mathrm{X}$-pander measurement without additional reaming. In $50.0 \%(n=39)$ of the procedures the orthopaedic surgeons answered that using the X-pander led to better cup insertion compared to trial cup fitting. In the remaining cases no difference to regular trial fitting was reported. In $37.2 \%$ of primary THA cases $(n=29)$ the surgeons stated that use the $\mathrm{X}$-pander could lead to the use of less additional screw fixation. In $48(61.5 \%)$ of cases the surgeon felt more confident in achieving press-fit by the use of the X-pander. The X-pander was not judged as time-saving in most of the cases, only 18 (23.1\%) judged it as being potentially time-saving. In 50 cases $(64.1 \%)$ the surgeon judged the X-pander suitable for replacing the traditional trial cup. 
In the revision cases ( 31 cases by 18 surgeons) the surgeon judged the X-pander as giving fairly to much more reliable information than traditional trial cups in $78.9 \%$ of the procedures. In 10 cases $(32.2 \%)$ the use of the $\mathrm{X}$-pander changed the decision about reaming intra operatively or the size of the definitive implant changed based on the X-pander measurement. In four (12.9\%) procedures this was a combination of further reaming and change of cup-size, in one $(3.2 \%)$ procedures only additional reaming was done without change of cup size and in five $(16.1 \%)$ cases only the size of the definitive implant was changed based on the X-pander measurement without additional reaming. The orthopaedic surgeons scored in 16 of the revision cases $(51.6 \%)$ that using the X-pander led to better cup insertion compared to trial cup fitting, in the other cases no to moderate difference compared to regular trial fitting was reported. In $25.8 \%$ of the revision cases $(n=8)$ the surgeons stated that using the X-pander could lead to the use of less additional screw fixation. In $18(58.1 \%)$ of the cases the surgeon felt more confident in achieving press-fit by the use of the X-pander. The X-pander was not judged as time-saving in most of the cases, only in six procedures $(19.4 \%)$ the orthopaedic surgeons judged it as being potentially time-saving. In 17 cases $(54.8 \%)$ the surgeon judged the $\mathrm{X}$-pander suitable for replacing the traditional trial cup.

Mixed logistic regression analysis showed no significant association between procedure (primary of revision), bone quality and experience of the surgeon on decision change concerning cup size, reaming or confidence regarding cup insertion and size.

\section{DISCUSSION}

The results of this survey on initial experience by the X-pander by orthopaedic surgeons show that this product may play a role in future hip surgery. Accordingly, surgeons stated that the X-pander give fairly to much more reliable information than traditional trial cups in $89.7 \%$ and $78.9 \%$ of respectively the primary and revision cases. Even more important is the fact that in primary cases $33.3 \%$ the choice of implant size changed, or further reaming was done and for revision cases this was $32.2 \%$ based on the X-pander measurement. Although no comparable literature is available about this device, it shows that the $\mathrm{X}$-pander is a feasible option to overcome problems regarding primary stability of the cup in THA.

Giving some surgeons the option to perform more procedures using the $\mathrm{X}$-pander and measure their experience, could introduce a bias. However, not every procedure was judged the same by these surgeons, since many factors for achieving press-fit are patient specific (6). To avoid bias by differences in the number of procedures performed by each surgeon, only the first five procedures per surgeon were included for analysis.

Although the definitive cup size changed in 30 of the 109 cases overall $(27.5 \%)$ when using the X-pander as measuring device, accounting 21 primary and nine revision cases, this does not mean that the decision to change the cup size is per definition better, since when surgeons are doubting about primary stability and choosing a bigger implant, per definition more press-fit is obtained by a larger cup. In this cases the X-pander gave the information that a bigger size fits and the fear for fractures on inserting the cup is not higher (9). Since many surgeons in this study used additional screws to ensure primary fixation it seems that in regular practice it remains a problem to achieve press-fit by the implant itself. Our outcomes that in 33.3\% of the primary cases and $32.2 \%$ of the revision cases the surgeon decided to change the cup size after measuring with the X-pander, show that the $\mathrm{X}$-pander can be a potential solution for the problem of achieving adequate press-fit without additional screw fixation (10-12).

\section{CONCLUSION}

This study validates that the X-pander may be a suitable option for accurate sizing and assessment of the reamed acetabulum and could replace traditional trial cups in THA.

\section{REFERENCES}

1. Haverkamp D, Westerbos S, Campo MM, et al. Early loosening of a press-fit cup with ceramic-on-ceramic 
articulation : our early results. Arch Orthop Trauma Surg. $2013 ; 133: 1757-1762$.

2. Henyš P, Čapek L. Impact Force, Polar Gap and Modal Parameters Predict Acetabular Cup Fixation : A Study on a Composite Bone. Ann Biomed Eng. 2018 ; 46 : 590-604.

3. Kuhn A, Scheller G, Schwarz M. Primary stability of cement-free press-fit acetabulum cups. In vitro displacement studies. Biomed Tech (Berl). 1999 ; 44 : 356-9

4. den Hartog YM, Mathijssen NMC, Vehmeijer SBW. The Less Invasive Anterior Approach for Total HIP Arthroplasty: A Comparison to other Approaches and an Evaluation of the Learning Curve - A Systematic Review. HIP Int. 2016 ; 26 : 105-120.

5. Brulc U, Antolič V, Mavčič B. Risk factors for unsuccessful acetabular press-fit fixation at primary total hip arthroplasty. Orthop Traumatol Surg Res. 2017 ; 103 : 993-997.

6. García-Rey E, García-Cimbrelo E, Cruz-Pardos A. Cup Press Fit in Uncemented THA Depends on Sex, Acetabular Shape, and Surgical Technique. Clin Orthop Relat Res. 2012 ; 470 : 3014-3023.

7. García-Cimbrelo E, García-Rey E. Bone Defect Determines Acetabular Revision Surgery. HIP Int. 2014 ; 24 : 33-36.
8. Hoornenborg D, de Waard S, Sierevelt IN, Haverkamp D. Dynamic trial fitting of the cup, does pre-expansion causes loss of primary stability (a biomechanical study). Hip International. 2018 ; 28, Issue 1, suppl, Euro-pean Hip Society 13th Congress

9. van Ladesteijn $\mathrm{R}$, Leslie $\mathrm{H}$, Manning WA, et al. Mechanical properties of cancellous bone from the acetabulum in relation to acetabular shell fixation and compared with the corresponding femoral head. Med Eng Phys. 2018 ; $53: 75-81$.

10. García-Rey E. Screws Are Not Needed when Secure Interference Fit of Uncemented Acetabular Components is Adequate : A 5- to 15-Year Follow-up with Clinical and Radiological Analysis. HIP Int. 2017 ; 27 : 267-272.

11. Yin X, Zhou Y, Tang Q, et al. Screw-Hole Clusters in Acetabular Cups: A Morphological Study of Optimal Positioning of Screw-Holes. HIP Int. 2017 ; 27 : 382-388.

12. Liu Q, Zhou Y, Xu H, et al. Safe Zone for Transacetabular Screw Fixation in Prosthetic Acetabular Reconstruction of High Developmental Dysplasia of the Hip. J Bone Jt Surgery-American. 2009 ; Vol 91 : 2880-2885. 